\title{
Predicting the Severity of Acute Urinary Toxicity after Brachytherapy with Iodine-125 for Localized Prostate Cancer
}

\author{
Ken Takeda, ${ }^{1}$ Keichi Jingu, ${ }^{1}$ Masashi Koto, ${ }^{1}$ Keisuke Fujimoto, ${ }^{1}$ \\ Kakutaro Narazaki, ${ }^{1}$ Masaki Kubozono, ${ }^{1}$ Hideo Saito, ${ }^{2}$ Shigeyuki Yamada, ${ }^{2}$ \\ Kohji Mitsuduka, ${ }^{2}$ Shigeto Ishidoya, ${ }^{2}$ Hisanori Ariga, ${ }^{1}$ Yoichi Arai ${ }^{2}$ and \\ Shogo Yamada ${ }^{1}$ \\ ${ }^{1}$ Department of Radiation Oncology, Tohoku University School of Medicine, Sendai, Japan \\ ${ }^{2}$ Department of Urology, Tohoku University School of Medicine, Sendai, Japan
}

\begin{abstract}
Prostate cancer is one of the common cancers in the world. In Japan, prostate brachytherapy (PB) with iodine-125 has become a treatment option for localized prostate cancer since 2003 . Nevertheless, severe acute urinary toxicity (AUT) remains as one of the intractable side effects. We assessed AUT and the changes in international prostate symptom score (IPSS) before and after PB for localized prostate cancer. IPSS is a questionnaire tool for tracking the subjective urinary symptoms. Between 2006 and 2009, 104 eligible patients underwent PB with iodine-125 were analyzed. AUT was graded with the radiation therapy oncology group (RTOG) scale. Eligible patients filled out IPSS questionnaires before and after PB. Clinical and treatment-related factors were examined for correlation with the severity of AUT and the interval to IPSS resolution. AUT of RTOG Grade 0 (no changes) and Grade 2 was detected in one and 96 patients, respectively, whereas seven patients $(6.7 \%)$ experienced AUT of Grade 3. Thus, the incidence of severe AUT (Grade 3) after PB was low. A greater number of needles $(p=0.012)$ were associated with AUT of RTOG Grade 3 on the univariate analysis. The median interval to IPSS resolution was 6 months $(7 \pm 6$ months). Greater post-implant maximal IPSS $(p<0.001)$ was associated with slower IPSS resolution, whereas higher pre-implant IPSS $(p<0.001)$ was associated with faster IPSS resolution on the multivariate analysis. In conclusion, reducing the number of needles in PB may be helpful for decreasing the rate of severe AUT.
\end{abstract}

Keywords: prostate cancer; brachytherapy; iodine-125; acute urinary toxicity; quality of life

Tohoku J. Exp. Med., 2011, 223 (1), 55-60. C 2011 Tohoku University Medical Press

Prostate cancer is one of the common cancers in the world. The spread of prostate-specific antigen (PSA) screening has led to an increase in incidence and an increasing proportion of early, good-prognosis prostate cancers (Ferrer et al. 2008). Prostate brachytherapy (PB) is a standard treatment option for localized prostate cancer (LPC) because of its excellent long-term disease control and ability to conserve a patient's quality of life due to the relative absence of severe long-term side effects (Grimm et al. 2001; Potters et al. 2005). In Japan, PB with iodine- 125 has become a treatment option for LPC since 2003. Nevertheless, severe acute urinary toxicity (AUT) such as irritative voiding symptoms and occasional prolonged catheterisation remain as common side effects (Bittner et al. 2007). Therefore, it is important to assess severe AUT and clarify the clinical and treatment factors related to severe AUT (Wallner et al. 2002; Beekman et al. 2005; Niehaus et al. 2006; Bittner et al. 2007). In addition, the inclusion of patient-reported toxicity data was reported to provide more detailed assessment of AUT (Namiki et al. 2006; Ash et al. 2007; Ferrer et al. 2008). Therefore, AUT after PB was examined using the radiation therapy oncology group (RTOG) scale as an objective parameter and the international prostate symptom score (IPSS) as a subjective variable in this study. IPSS was used to screen for diagnosis and tracking the subjective urinary symptoms of the benign prostatic hyperplasia (Bosch et al. 1995). In the present study, we describe the incidence and associated factors for AUT of RTOG Grade 3 and IPSS resolution as a surrogate for recovery from acute subjective urinary symptoms.

\section{Patients and Methods}

Eligibility criteria for $P B$

Eligible patients included those with low-risk disease (clinical stage T2a or lower, initial prostate specific antigen [iPSA] level $\leq 10.0 \mathrm{ng} / \mathrm{mL}$ and Gleason score $(\mathrm{GS}) \leq 6$ ), and "low-tier" intermedi-

Received July 12, 2010; revision accepted for publication December 14, 2010. doi: 10.1620/tjem.223.55

Correspondence: Ken Takeda, Department of Radiation Oncology, Tohoku University School of Medicine, 1-1, Seirho-machi, Aoba-ku, Sendai, 980-8574, Japan.

e-mail: takedak41@yahoo.co.jp 
ate-risk disease (stage T2b or lower, iPSA $10-20 \mathrm{ng} / \mathrm{mL}$, and GS $\leq 7$ ) (Barrett et al. 2009). Patients with low-risk prostate cancer and a prostate volume (PV) of $\leq 40 \mathrm{~cm}^{3}$ were treated with $\mathrm{PB}$ alone. Patients with GS $=7$ or PV larger than $40 \mathrm{~cm}^{3}$ received neoadjuvant hormonal therapy (NHT) before PB to reduce PV.

We treated 130 patients with localized prostate cancer with iodine-125 permanent seed implantation at our institution between July 20, 2006 and June 1, 2009. Among the 130 patients, 21 patients who did not have a minimal follow-up of 6 months nor sufficient serial IPSS data at least three subsequent assessments after PB were not included in this analysis. In addition, the other five patients were excluded from the analysis because they did not fulfil one component of our eligibility criteria. Therefore, we analysed 104 patients in this study.

Written informed consent was obtained from each patient before PB. The research ethics board of Tohoku University School of Medicine approved this study.

\section{Implant procedure}

The pre-implant treatment planning was performed to assess pre-implant ultrasound PV (PUPV) about 1 month before the implant procedure. The patient was placed in the extended lithotomy position for the procedure. Implants were designed from transrectal ultrasound (TRUS) images of the prostate taken at 5-mm intervals from the base through the apex. The captured images were digitised with a planning computer using the VariSeed 7.1 planning system (Varian Medical Systems, Palo Alto, CA, USA). The clinical target volume was defined as the prostate visualised on the TRUS images. A prescribed dose of 145 Gy was designed to cover $\geq 95 \%$ of the planning target volume (PTV) (prostate with 3-5-mm margins). Using a peripheral loading technique, we set the treated volume to include the PTV within the prescribed isodose. The implantation was performed via TRUS guidance with the patient under spinal anaesthesia and in the extended lithotomy position with an aerated jelly in the urethral catheter allowed for identification of the urethra. A Mick applicator (Mick Radionuclear Instruments, Bronx, NY, USA) was used to deposit the seeds. I-125 was the only source used, with a mean activity of $0.34 \mathrm{mCi} /$ seed (range, $0.29-0.40 \mathrm{mCi} / \mathrm{seed}$ ). To minimise micturition problems, an alpha-blocker (silodosin, $8 \mathrm{mg} /$ day, orally) was routinely prescribed to all patients from the day after implantation, and it was continued until it was no longer required symptomatically.

Computed tomography (CT) scans were obtained 1 day and 1 month after implantation for post-implant dosimetric analysis. Axial CT scan images of the pelvis were taken at 1-mm intervals. The PB radiation oncologist contoured the prostate and urethra carefully for each patient. The urethral dose was defined by doses at the centre of the prostate on $\mathrm{CT}$ images acquired 1 month after PB. The calculated dosimetry parameters were the percentage of prostate volume receiving $100 \%, 150 \%$, and $200 \%$ of the prescribed minimal peripheral dose (prostate $\mathrm{V}_{100}, \mathrm{~V}_{150}$, and $\mathrm{V}_{200}$, respectively) and the minimal dose values received by $90 \%$ of the PV (prostate $\mathrm{D}_{90}$ ). Additionally, the minimal dose values received by $90 \%$ of the urethra volume (urethral $\mathrm{D}_{90}$ ) were recorded.

\section{Outcome measurements}

Clinical follow-up was started from the day of implantation. Current eligible patients filled out self-assessment IPSS questionnaires at pre-implant IPSS (as IPSS-baseline), and at 1, 3, 6, 12, and 24 months after treatment. The minimum IPSS follow-up for this study group was 6 months. Post-implant maximal IPSS (IPSS-max) was also recorded as the highest score for acute urinary symptoms within 3 months after PB. IPSS resolution was defined as a return of IPSS-max to within 2 points of the IPSS-baseline score.

\section{Assessment of AUT}

AUT was graded using the RTOG scale (Lawton et al. 1991). According to that scale, AUT was defined as morbidity occurring within 3 months after seed implantation. The RTOG scale of AUT is as follows:

RTOG Grade 0: no change.

RTOG Grade 1: frequency of urination or nocturia twice the pre-treatment habit; dysuria; urgency not requiring medication.

RTOG Grade 2: frequency of urination or nocturia less frequent than every hour. Dysuria, urgency, or bladder spasm requiring a local anesthetic (e.g., Pyridium).

RTOG Grade 3: frequency with urgency and nocturia hourly or more frequently; dysuria, pelvic pain, or bladder spasm requiring regular, frequent narcotics; gross hematuria with or without clot passage; obstruction requiring an indwelling catheter or a minor procedure.

RTOG Grade 4: hematuria requiring transfusion; acute bladder obstruction not secondary to clot passage; ulceration or necrosis.

\section{Statistical analyses}

Clinical, treatment-related, and dosimetric factors were assessed for univariate and multivariate correlations with acute urinary RTOG Grade 3 or worse toxicities and the interval to IPSS resolution. Clinical and treatment-related factors included patient age, iPSA level, diabetes, hypertension, the utilisation of NHT, presence of acute urinary retention, IPSS-baseline, IPSS-max, PUPV, CT-determined PV 1 day (CTPV1D) and 1 month after implant (CTPV1M), the ratio between CTPV1D and CTPV1M as a surrogate of post-implant oedema, and the number of needles and seeds used. Dosimetric factors included prostate $\mathrm{V}_{100}, \mathrm{~V}_{150}, \mathrm{~V}_{200}$, prostate $\mathrm{D}_{90}$, and urethral $\mathrm{D}_{90}$.

The interval to IPSS resolution was examined using KaplanMeier curves. We assessed the relationship between the interval to IPSS resolution and those factors using the log-rank test and MannWhitney $U$-test for the univariate analysis (UVA) and a Cox proportional hazard regression analysis for the multivariate analysis (MVA). All factors were input to the MVA. Furthermore, the correlation between acute urinary RTOG Grade 3 or worse toxicities and these variables excluding acute urinary retention was evaluated. Because acute urinary retention was considered to be one of the acute urinary Grade 3 or worse toxicities, the chi-square test and Mann-Whitney $U$-test were used in the UVA. The logistic regression test was performed for the MVA. These analyses were conducted using SPSS 11.0 (SPSS, Chicago, IL, USA). Differences were regarded as statistically significant at $P$-values less than 0.05 .

\section{Results}

The patient characteristics are shown in Table 1. The median follow-up period was 21 months (range, 6-36 months). No patient received supplementary external beam radiotherapy or adjuvant hormonal therapy.

\section{AUT of RTOG Grade 3 and acute urinary retention AUT of RTOG Grade 0 and AUT of Grade 1-2 were}


Table 1. Patient characteristics.

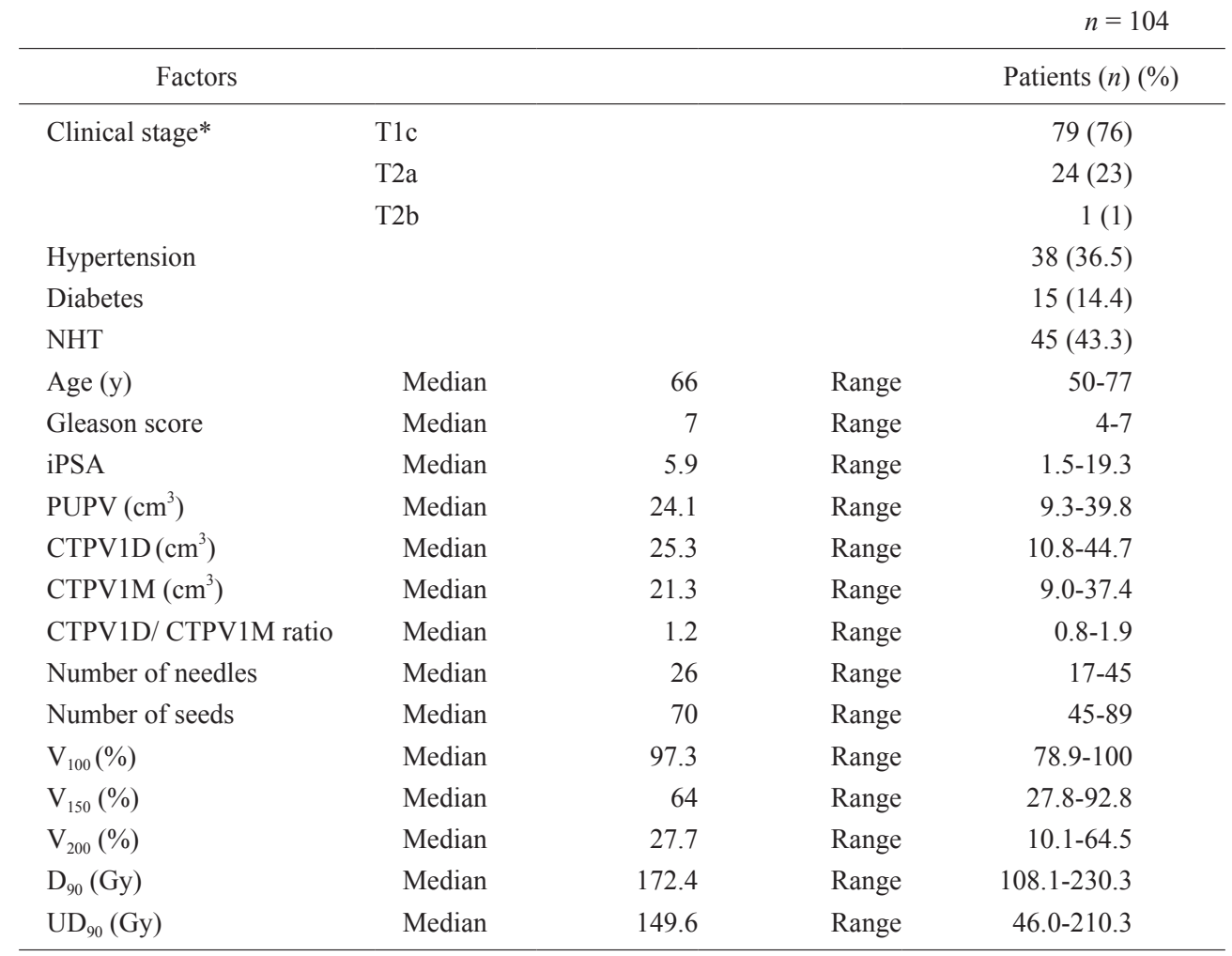

NHT, neoadjuvant hormonal therapy; iPSA, initial prostate-specific antigen; PUPV, preimplant ultrasound prostate volume; CTPV1D, computed tomography determined prostate volume 1 day after implant; CTPV1M, computed tomography determined prostate volume 1 month after implant; $\mathrm{V}_{100}, \mathrm{~V}_{150}, \mathrm{~V}_{200}$, percentage of prostate volume receiving $100 \%, 150 \%$, and $200 \%$ of the prescribed minimal peripheral dose, respectively; $\mathrm{D}_{90}$, minimal dose received by $90 \%$ of the prostate; $\mathrm{UD}_{90}$, minimal dose received by $90 \%$ of the urethra. *2002 TNM staging system.

recorded in one and 96 patients, respectively. AUT of RTOG Grade 3 was recorded in seven patients (6.7\%). Of these seven patients, five experienced acute urinary retention, and two had other urinary symptoms. However, they did not need surgical intervention for the symptoms. Thus, none of the patients experienced AUT of RTOG Grade 4. A greater number of needles $(p=0.012)$ were associated with AUT of RTOG Grade 3 on the UVA, but not on the MVA (Table 2).

\section{IPSS resolution}

The IPSS increased and peaked by the 3-month visit after $\mathrm{PB}$, but subsequently recovered to the baseline level. The median IPSS-baseline and IPSS-max were 7 (range, 0-23) and 17 (range, 0-34), respectively. The IPSS-max in one patient was 0 . Of the 104 patients, IPSS resolution was achieved in 92 patients $(88.5 \%$ ) (Fig. 1). The median interval to IPSS resolution was 6 months $(7 \pm 6$ months $)$. A greater IPSS-max $(p<0.001)$ was associated with a slower IPSS resolution in the MVA. A greater IPSS-baseline $(p<$ 0.001 ) resulted in faster IPSS resolution (Table 3). The relationship between PUPV and IPSS resolution was equivocal.

\section{Discussion}

Our incidence of AUT at RTOG Grade 3 was $6.7 \%$, which was comparatively lower than that in other reports. The reason may be due to the difference of PV. Keyes et al. (2009) reported an incidence of AUT at RTOG Grade 3 of $16.2 \%$. Their median of planning ultrasound-determined target volume was $38.0 \mathrm{~cm}^{3}$ (range, $17.0-67.2 \mathrm{~cm}^{3}$ ). According to Williams et al. (2004), the incidence of acute urinary retention was $19.7 \%$ and the PV in TRUS was 35.1 $\pm 8.6 \mathrm{~cm}^{3}$. Salem et al. (2003) reported that 11 among 60 consecutive patients developed AUT at Grade 3 and their median pre-implant prostate ultrasound volume was $30 \mathrm{ml}$. Wust et al. (2004) found that $14 \%$ of their patients required a catheter because of manifest retention and the PV was 34 $\pm 14 \mathrm{ml}$. In addition, according to Keyes et al. (2009), a larger pre-implant PV increased the likelihood of acute RTOG Grade 3 toxicity. Our median of PV was $24.1 \mathrm{~cm}^{3}$ and was smaller compared with previous studies, as summarized in Table 1. Such a difference in PV may account for the lower rate of severe AUT in our patients.

In our study, only the number of needles used was significantly associated with AUT of RTOG Grade 3 in the UVA, although we could not find a relationship between 
Table 2. Factors associated with acute urinary RTOG Grade 3 toxicity after prostate brachytherapy.

\begin{tabular}{lll}
\hline \multicolumn{1}{c}{ Factors } & UVA & MVA \\
& $p$ & $p$ \\
\hline Age & 0.775 & 0.855 \\
iPSA & 0.312 & 0.275 \\
Diabetes & 0.991 & 0.187 \\
Hypertension & 0.65 & 0.195 \\
NHT & 0.982 & 0.624 \\
IPSS-baseline & 0.219 & 0.504 \\
IPSS-max & 0.071 & 0.158 \\
PUPV & 0.504 & 0.183 \\
CTPV1D $\left(\mathrm{cm}^{3}\right)$ & 0.636 & 0.392 \\
CTPV1M $\left(\mathrm{cm}^{3}\right)$ & 0.631 & 0.317 \\
CTPV1D/CTPV1M ratio & 0.731 & 0.431 \\
Number of needles & 0.012 & 0.1 \\
Number of seeds & 0.824 & 0.583 \\
$\mathrm{~V}_{100}$ & 0.496 & 0.917 \\
$\mathrm{~V}_{150}$ & 0.751 & 0.212 \\
$\mathrm{~V}_{200}$ & 0.581 & 0.45 \\
$\mathrm{D}_{90}$ & 0.82 & 0.231 \\
UD $_{90}$ & 0.086 & 0.148 \\
\hline
\end{tabular}

IPSS, International Prostate Symptom Score; UVA, univariate analysis; MVA, multivariate analysis; CI, confidence interval; other abbreviations as in Table 1.
AUT of RTOG Grade 3 and the number of seeds. Probably the number of our patients included in the analysis is too low to find the number of seeds as predictor. Several authors also reported a correlation between AUT and number of needles. Wust et al. (2004) demonstrated a relationship between the number of needles and urinary toxicity in the acute phase. According to Keyes et al. (2009), a greater number of needles contributed to acute RTOG Grade 2 or

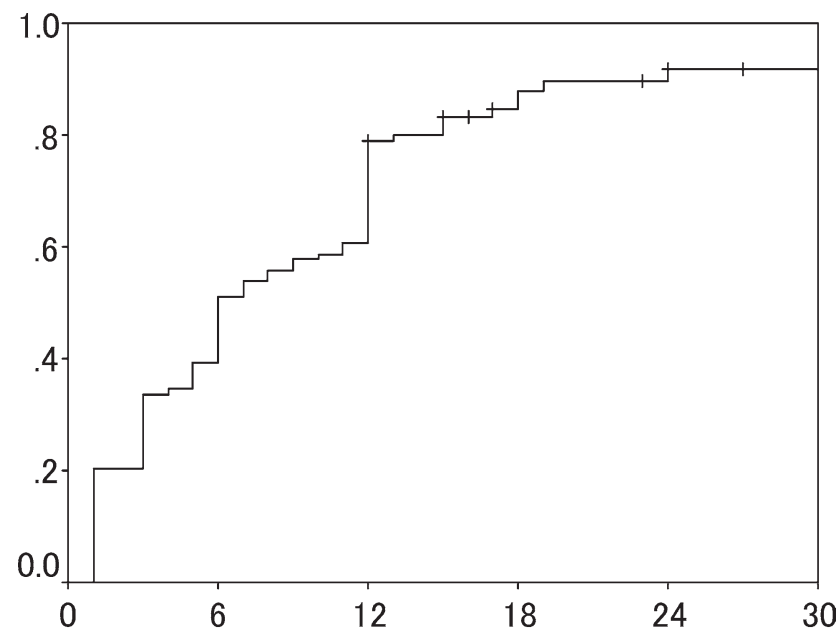

Months

Fig. 1. Kaplan-Meier curve of the interval to international prostate symptom score (IPSS) resolution in 104 patients. IPSS resolution was achieved in 92 patients $(88.5 \%)$.

Table 3. Factors associated with the interval to IPSS resolution after prostate brachytherapy.

\begin{tabular}{|c|c|c|c|}
\hline \multirow{2}{*}{ Factors } & \multirow{2}{*}{$\begin{array}{c}\text { UVA } \\
\qquad p\end{array}$} & \multicolumn{2}{|r|}{ MVA } \\
\hline & & $P$ & Odds ratio $(95 \% \mathrm{CI})$ \\
\hline Age & 0.173 & 0.141 & - \\
\hline iPSA & 0.649 & 0.45 & - \\
\hline Diabetes & 0.588 & 0.314 & - \\
\hline Hypertension & 0.654 & 0.967 & - \\
\hline NHT & 0.397 & 0.15 & - \\
\hline AUR & 0.594 & 0.676 & - \\
\hline IPSS-baseline & 0.024 & $<0.001$ & $1.16(1.097-1.226)$ \\
\hline IPSS-max & 0.001 & $<0.001$ & $0.883(0.847-0.921)$ \\
\hline PUPV & 0.295 & 0.019 & $1.11(1.02-1.211)$ \\
\hline CTPV1D $\left(\mathrm{cm}^{3}\right)$ & 0.11 & 0.74 & - \\
\hline CTPV1M $\left(\mathrm{cm}^{3}\right)$ & 0.242 & 0.93 & - \\
\hline CTPV1D/CTPV1M ratio & 0.344 & 0.823 & - \\
\hline Number of needles & 0.789 & 0.57 & - \\
\hline Number of seeds & 0.053 & 0.054 & - \\
\hline $\mathrm{V}_{100}$ & 0.776 & 0.995 & - \\
\hline $\mathrm{V}_{150}$ & 0.675 & 0.684 & - \\
\hline $\mathrm{V}_{200}$ & 0.68 & 0.653 & - \\
\hline $\mathrm{D}_{90}$ & 0.534 & 0.983 & - \\
\hline $\mathrm{UD}_{90}$ & 0.796 & 0.957 & - \\
\hline
\end{tabular}

AUR, acute urinary retention; other abbreviations as in Table 1 and Table 2. 
worse toxicity, whereas it was not significantly associated with acute RTOG Grade 3 toxicity.

Our median interval to IPSS resolution was 6 months and shorter than several reports (Williams et al. 2004; Keyes et al. 2009), or comparable to others (Ohashi et al. 2006; Bottomley et al. 2007; Anderson et al. 2009). The short interval may be associated with our lower incidence of severe AUT, compared with the findings of other reports (Williams et al. 2004; Keyes et al. 2009). In addition, we revealed that a greater IPSS-baseline was related to a faster IPSS resolution, and a greater IPSS-max was associated with a slower IPSS resolution, consistent with the previous studies (Neill et al. 2007; Keyes et al. 2009). According to Neill et al. (2007), one reason that a higher IPSS-baseline predicts a faster IPSS resolution may be that the higher the IPSS-baseline is, the smaller is the range of IPSS increase required to reach IPSS-max due to the maximal IPSS score of 35 points. Those patients with a higher baseline score may tend to recover to that value faster than those with a lower baseline. According to Keyes et al. (2009), this might be related to patient expectations and/or to the preexisting urinary symptoms, which might mask any new dysfunction caused by PB.

Although several authors reported relationship between dosimetric variables and IPSS normalization (Wust et al. 2004; Neill et al. 2007), we found no correlation between acute urinary morbidity and prostate $\mathrm{V}_{100}, \mathrm{~V}_{150}$, $\mathrm{V}_{200}$, or $\mathrm{D}_{90}$. One reason may be that the PV in this analysis is too small to find correlation between dosimetric parameters, AUT at RTOG Grade 3, and IPSS resolution. Compared to our study, PV in their study was greater, and the prostate size difference may have contributed to differences in findings.

We found no relationship between urethral dose and AUT of Grade 3, similar to several reports (Allen et al. 2005; Ohashi et al. 2006; Neill et al. 2007). In contrast, Thomas et al. (2008) reported that a greater urethral dose at the prostate base predicted worse urinary toxicity after PB, and variation in the urethral dose may have had a significant direct effect on toxicity at the prostate base. Wallner et al. (1995) also demonstrated increased urinary RTOG Grade morbidity with exceptionally high urethral doses. They reported that patients with RTOG Grades $0-1$ or urinary morbidity Grades 2-3 had average maximum urethral doses of 447 Gy and 592 Gy, respectively (Wallner et al. 1995; Thomas et al. 2008). In the present study, the assessment of urethral catheter placement for collecting dosimetric data was not performed during CT 1 month after $\mathrm{PB}$. We might not have accurately contoured the urethral wall and may have failed to archive adequate urethral dosimetric data in several patients, which may have caused the absence of a statistical relationship between urethral dose and urinary morbidity. This was one of our limitations in this study.

Our study had several limitations, elsewhere. First, we did not analyze a more detailed relationship between uri- nary dose and urinary morbidity. In contrast, Williams et al. (2004) reported that a heightened IPSS change was correlated to an increased number of seeds implanted above the level of the prostate base. Second, multiple urinary evaluations using measures such as a combination of IPSS, University of California at Los Angeles Prostate Cancer Index, and the Expanded Prostate Index Composite urinary scores, should be considered to provide more objective measurement, whereas we used only the IPSS and the acute RTOG scale to assess acute urinary symptoms in this study (Namiki et al. 2006; Ash et al. 2007). Several authors have demonstrated that patient-reported toxicity data are superior to physician-reported data (Namiki et al. 2006; Ash et al. 2007; Ferrer et al. 2008). Third, this study was limited to acute phase toxicity after PB. A longer follow-up and further study are necessary to estimate long term treatment outcomes and toxicities. Forth, we could not mention the duration of NHT in all patients. Several patients in this study had received NHT at the other hospitals before PB. There was no detailed information of their duration of NHT.

\section{Conclusion}

Our incidence of severe AUT after PB was comparatively lower than previous reports. Mostly subjective acute urinary symptom after PB recovered within around 1 year. Worse acute urinary symptom after PB appears to slow the improvement, whereas severer urinary symptom before $\mathrm{PB}$ may hasten the recovery. A greater number of needles are associated with severe AUT. Therefore, we may be able to decrease the rate of severe AUT by reducing number of needles in PB.

\section{References}

Allen, Z.A., Merrick, G.S., Butler, W.M., Wallner, K.E., Kurko, B., Anderson, R.L., Murray, B.C. \& Galbreath, R.W. (2005) Detailed urethral dosimetry in the evaluation of prostate brachytherapy-related urinary morbidity. Int. J. Radiat. Oncol. Biol. Phys., 62, 981-987.

Anderson, J.F., Swanson, D.A., Levy, L.B., Kuban, D.A., Lee, A.K., Kudchadker, R., Phan, J., Bruno, T. \& Frank, S.J. (2009) Urinary side effects and complications after permanent prostate brachytherapy: the MD Anderson Cancer Center Experience. Urology, 74, 601-605.

Ash, D., Bottomley, D., Al-Qaisieh, B., Carey, B., Gould, K. \& Henry, A. (2007) A prospective analysis of long-term quality of life after permanent I-125 brachytherapy for localized prostate cancer. Radiother. Oncol., 84, 135-139.

Barrett, A., Dobbs, J., Morris, S. \& Roques, T. (2009) Practical Radiotherapy Planning, 4th ed., Hodder Arnold, An Hachette UK Company.

Beekman, M., Merrick, G.S., Butler, W.M., Wallner, K.E., Allen, Z.A. \& Galbreath, R.W. (2005) Selecting patients with pretreatment postvoid residual urine volume less than $100 \mathrm{~mL}$ may favorably influence brachytherapy-related urinary morbidity. Urology, 66, 1266-1270.

Bittner, N., Merrick, G.S., Wallner, K.E., Lief, J.H., Butler, W.M. \& Galbreath, R.W. (2007) The impact of acute urinary morbidity on late urinary function after permanent prostate brachytherapy. Brachytherapy, 6, 258-266.

Bosch, J.L., Hop, W.C., Kirkels, W.J. \& Schröder, F.H. (1995) Natural history of benign prostatic hyperplasia: appropriate 
case definition and estimation of its prevalence in the community. Urology, 46, 34-40.

Bottomley, D., Ash, D., Al-Qaisieh, B., Carey, B., Joseph, J., St Clair, S. \& Gould, K. (2007) Side effects of permanent I 125 prostate seed implants in 667 patients treated in Leeds. Radiother. Oncol., 82, 46-49.

Ferrer, M., Suárez, J.F., Guedea, F., Fernández, P., Macías, V., Mariño, A., Hervas, A., Herruzo, I., Ortiz, M.J., Villavicencio, H., Craven-Bratle, J., Garin, O. \& Aguiló, F.; Multicentric Spanish Group of Clinically Localized Prostate Cancer. (2008) Health-related quality of life 2 years after treatment with radical prostatectomy, prostate brachytherapy, or external beam radiotherapy in patients with clinically localized prostate cancer. Int. J. Radiat. Oncol. Biol. Phys., 72, 421-432.

Grimm, P.D., Blasko, J.C., Sylvester, J.E., Meier, R.M. \& Cavanagh, W. (2001) Ten-year biochemical (prostate-specific antigen) control of prostate cancer with (125)I brachytherapy. Int. J. Radiat. Oncol. Biol. Phys., 51, 31-40.

Keyes, M., Miller, S., Moravan, V., Pickles, T., McKenzie, M., Pai, H., Liu, M., Kwan, W., Agranovich, A., Spadinger, I., Lapointe, V., Halperin, R. \& Morris, W.J. (2009) Predictive factors for acute and late urinary toxicity after permanent prostate brachytherapy: long-term outcome in 712 consecutive patients. Int. J. Radiat. Oncol. Biol. Phys., 73, 1023-1032.

Lawton, C.A., Won, M., Pilepich, M.V., Asbell, S.O., Shipley, W.U., Hanks, G.E., Cox, J.D., Perez, C.A., Sause, W.T., Doggett, S.R. \& Rubin, P. (1991) Long-term treatment sequelae following external beam irradiation for adenocarcinoma of the prostate: Analysis of RTOG studies 7506 and 7706. Int. J. Radiat. Oncol. Biol. Phys., 21, 935-939.

Namiki, S., Satoh, T., Baba, S., Ishiyama, H., Hayakawa, K., Saito, S. \& Arai, Y. (2006) Quality of life after brachytherapy or radical prostectomy for localized prostate cancer: a prospective longitudinal study. Urology, 68, 1230-1236.

Neill, M., Studer, G., Le, L., McLean, M., Yeung, I., Pond, G. \& Crook, J.M. (2007) The nature and extent of urinary morbidity in relation to prostate brachytherapy urethral dosimetry. Brachytherapy, 6, 173-179.

Niehaus, A., Merrick, G.S., Butler, W.M., Wallner, K.E., Allen,
Z.A., Galbreath, R.W. \& Adamovich, E. (2006) The influence of isotope and prostate volume on urinary morbidity after prostate brachytherapy. Int. J. Radiat. Oncol. Biol. Phys., 64, 136-143.

Ohashi, T., Yorozu, A., Toya, K., Saito, S. \& Momma, T. (2006) Serial changes of international prostate symptom score following I-125 prostate brachytherapy. Int. J. Clin. Oncol., 11, 320-325.

Potters, L., Morgenstern, C., Calugaru, E., Fearn, P., Jassal, A., Presser, J. \& Mullen, E. (2005) Twelve-year outcomes following permanent prostate brachytherapy in patients with clinically localized prostate cancer. J. Urol., 173, 1562-1566.

Salem, N., Simonian-Sauve, M., Rosello, R., Alzieu, C., Gravis, G., Maraninchi, D. \& Bladou, F. (2003) Predictive factors of acute urinary morbidity after iodine- 125 brachytherapy for localised prostate cancer: a phase 2 study. Radiother. Oncol., 66, 159-165.

Thomas, C., Keyes, M., Liu, M. \& Moravan, V. (2008) Segmental urethral dosimetry and urinary toxicity in patients with no urinary symptoms prior to permanent prostate brachytherapy. Int. J. Radiat. Oncol. Biol. Phys., 72, 447-455.

Wallner, K., Roy, J. \& Harrison, L. (1995) Dosimetry guidelines to minimize urethral and rectal morbidity following transperineal I-125 prostate brachytherapy. Int. J. Radiat. Oncol. Biol. Phys., 32, 465-471.

Wallner, K., Merrick, G., True, L., Cavanagh, W., Simpson, C. \& Butler, W. (2002) I-125 versus Pd-103 for low-risk prostate cancer: morbidity outcomes from a prospective randomized multicenter trial. Cancer. J., 8, 67-73.

Williams, S.G., Millar, J.L., Duchesne, G.M., Dally, M.J., Royce, P.L. \& Snow, R.M. (2004) Factors predicting urinary morbidity following 125 -iodine transperineal prostate brachytherapy. Radiother. Oncol., 73, 33-38.

Wust, P., Wischka von Borczyskowski, D., Henkel, T., Rosner, C., Graf, R., Tilly, W., Budach, V., Felix, R. \& Kahmann, F. (2004) Clinical and physical determinants for toxicity of 125-I seed prostate brachytherapy. Radiother. Oncol., 73, $39-48$. 